\title{
Implicaciones del turismo masivo en un destino de cruceros
}

\author{
Silvia Sanz-Blasa, Daniela Buzova ${ }^{a}$, Fernando Garrigós-Simón ${ }^{\mathrm{b}}$, Yeamduan \\ Narangajavana Kaosiri ${ }^{\mathrm{c}}$ \\ ${ }^{a}$ Departamento de Comercialización e Investigación de Mercados, Universitat de Valencia, Valencia, \\ España silvia.sanz@uv.es, daniela.buzova@uv.es, 'bepartamento de Organización de Empresas, \\ Universitat Politècnica de València, Valencia, España fgarrigos@doe.upv.es, 'CUniversitat Jaume I, \\ Castellón, España ynaranga@uji.es
}

\begin{abstract}
Resumen
Una de las tipologías turísticas que pueden generar mayores problemas de masificación turística es el turismo de cruceros, dada la gran cantidad de personas que desembarcan en un puerto de escala para la visita del destino. Por este motivo, el presente trabajo tiene por objetivo analizar la percepción de masificación turística del turista de cruceros que visita un importante puerto de escala del mediterráneo y como la misma influye en su satisfacción con la visita realizada. Para ello, usando modelos de ecuaciones estructurales, y a partir de una muestra de cruceristas, se testa el modelo propuesto en la presente investigación. Los resultados indican que de las dos tipologías de masificación turística analizadas (humana y espacial), es la masificación espacial la que contribuye en mayor medida a la percepción de masificación turística. Además, se observa que la percepción de masificación afecta a la satisfacción del turista, aunque su efecto en esta tipología de turistas es bastante débil. Los resultados sugieren implicaciones relevantes para los gestores turísticos del destinos.
\end{abstract}

Palabras clave: Masificación turística, satisfacción del visitante, modelos de ecuaciones estructurales, masificación humana, masificación espacial.

\footnotetext{
Abstract

One of the types of tourism that can generate greater problems of overtourism is cruise ship tourism, given the large number of people who disembark at a port of call to visit the destination. For this reason, the present study aims to analyze the perception of massification among cruise ship tourists visiting an important Mediterranean port of call and how it influences their satisfaction with their visit. To this end, using structural equation models and from a sample of cruise ship passengers, the model
} 
proposed in this study is tested. The results indicate that of the two typologies of massification analysed, it is spatial massification that contributes most to the perception of tourism massification. Furthermore, it is observed that the perception of massification affects tourist satisfaction, although the effect is quite weak. The results suggest relevant implications for destination managers.

Keywords: Overtourism, visitor satisfaction, structural equation models, human massification, spatial massification.

\section{Introducción}

Es destacable las elevadas tasas de crecimiento del turismo de cruceros en los últimos años, lo que hace necesario analizar con mayor profundidad el impacto global de este modelo de turismo. Según datos de la Asociación Internacional de Empresas de Cruceros (CLIA), este sector movilizó, en 2018, 7.17 millones de pasajeros, con un volumen de negocio de 4.250 millones de euros, generando un total de 33.000 empleos (CLIA, 2019).

Sin duda, los beneficios derivados de la práctica de este tipo de turismo son elevados, pero su desarrollo también presenta inconvenientes importantes. El hecho de que en cada visita a un puerto de escala desembarquen un elevado número de turistas genera importantes problemas, además de los asociados, cuando un buque llega a un puerto, con alteraciones del medio acuático, atmosférico y sonoro que pueden verse reflejadas en una degradación de los mismos. Así por ejemplo, la contaminación atmosférica, producida a través de partículas generadas en la combustión, puede provocar, además de la degradación del aire, degradación de las fachadas de los edificios históricos y otras infraestructuras.

La concentración de este tipo de turistas, al llegar varios miles de personas a la vez a un puerto de escala, provoca sensación de congestión del destino visitado, que hace que se relacione a los cruceros con el fenómeno denominado - vertourism" (Seraphin et al., 2018). Si bien, existe amplia literatura centrada en analizar la percepción de overtourism por parte de residentes (ver entre otros Cheung y Li, 2019; González et al., 2018; Kuščer y Mihalič, 2019; Smith et al., 2019), todavía son escasos los estudios que analizan dicha percepción por parte del turista que visita un destino (Sanz et al., 2019). Esta percepción del turista es especialmente relevante dado que puede afectar a la calidad de su experiencia, expectativas, satisfacción e incluso intenciones de comportamiento ((Sanz-Blas et al., 2019). 
Teniendo en cuenta lo anterior, el presente trabajo tiene por objetivo ampliar el ámbito de investigación llevado a cabo hasta el momento analizando si la percepción de masificación del turista de cruceros influye en su satisfacción con la visita realizada.

\section{Marco teórico}

El término masificación turística puede ser definido como una forma de sobrecarga de estímulo causada por contactos inapropiados o desconocidos con otras personas (Esser, 1972). Se refiere al nivel de saturación de un destino, excediéndose el número de personas que pueden a la vez visitar un destino, pudiendo causar destrucción del ambiente físico, económico y socio-cultural (Jurado et al., 2013).

Los turistas en su visita pueden percibir masificación turística no sólo negativa sino también positiva, pudiendose en parte explicar por los límites de tolerancia significativamente más altos que presentan algunos turistas (Popp, 2012). Se une a lo anterior, el interés que muestran por compartir experiencias y relacionarse con otras personas, observando a la gente o participando en grupos (Arnberger et al. 2010; Bryon \& Neuts 2008). Además, la literatura permite diferenciar entre dos tipos de masificación turística: la humana y la espacial (Kim et al., 2016). Los turistas perciben que un destino está espacialmente masificado cuando se sienten restringidos en sus movimientos. Por otro lado, perciben masificación humana cuando el número de personas en torno a ellos $\mathrm{y}$, por tanto, las interacciones sociales, son elevadas (Li et al., 2009).

Estudios previos han evidenciado que los turistas de cruceros que visitan un destino perciben tanto masificación positiva y negativa, como humana (cantidad de gente) y espacial (restricciones de movimiento) (Sanz-Blas et al., 2019), pudiendo dicha percepción condicionar el atractivo percibido del lugar, mermar la buena calidad de su experiencia, afectar a su satisfacción, valorando por debajo de lo esperado el destino visitado (Jurado et al., 2013; Li et al., 2017; Sanz-Blas et al., 2019).

La relación entre percepción de masificación turística y satisfacción todavía requiere de una mayor investigación científica, ya que presenta resultados contrapuestos en la literatura. De ese modo, es posible encontrar estudios que demuestran un efecto negativo significativo de la percepción de masificación sobre la satisfacción del turista (Zehrer y Raich, 2016). Otras investigaciones han evidenciado una relación nula entre ambas variables (Li et al., 2017). Por último, encontramos aquellos estudios que muestran una relación positiva entre masificación y satisfacción del turista (Díaz-Sauceda et al., 2015; Noone y Mattila, 2009). 
La falta de consenso en investigaciones previas en relación al efecto que la percepción de masificación tiene sobre la satisfacción del turista lleva, en la presente investigación, a formular la siguiente cuestiones a investigar:

¿La percepción de masificación turística influye en la satisfacción del turista de cruceros con la visita realizada al puerto de escala?

La figura 1 recoge las relaciones a contrastar en la presente investigación:

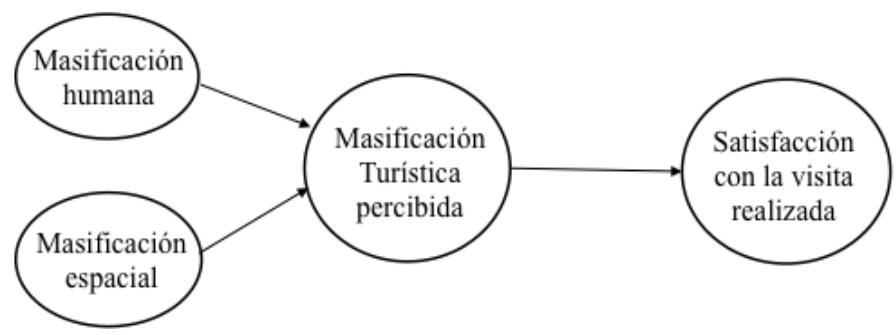

Fig. 1 Modelo teórico de la investigación

\section{Metodología}

La recogida de datos se realizó en uno de los principales puertos de recepción de turistas de cruceros del mediterráneo: Valencia, siendo el público objetivo los cruceristas que desembarcaron en la ciudad durante el último trimestre del año. Se utilizó la entrevista personal para recoger la información, utilizando un cuestionario estructurado traducido en diferentes idiomas: español, inglés, alemán e italiano. El total de cuestionarios correctamente cumplimentados fue de 467.

En la investigación, la masificación turística se concibió como un constructo formativo integrado por dos dimensiones: humana y espacial, siguiendo el estudio de Kim et al. (2016). Los tres ítems de la escala de satisfacción fueron tomados del estudio de Sanz-Blas y Carvajal-Trujillo (2014).

Para dar respuesta a la cuestión a investigar planteada en la presente investigación, se utilizó la técnica PLS de ecuaciones estructurales, utilizando el software Smart-PLS 3.1.

\section{Resultados}

Previamente a testar el modelo teórico de la presente investigación, se comprobó que los instrumentos de medida cumplían las propiedades psicométricas establecidas por la literatura. 
A continuación, se procedió a estimar el modelo estructural propuesto. Para ello, se obtuvieron los valores de la varianza explicada por el modelo (R2), los coeficientes path estandarizados $(\beta)$ con los valores $t$ observados obtenidos de la prueba bootstrap con 5000 submuestras (Hair et al., 2017). En la tabla 1 se muestran los resultados de la evaluación del modelo propuesto.

Tabla 1. Resultados del modelo estructural

\begin{tabular}{|l|c|c|c|c|}
\hline \multicolumn{1}{|c|}{ Hipótesis } & $\begin{array}{c}\text { Coeficiente } \\
\text { path }(\beta)\end{array}$ & Pesos & $\begin{array}{c}\text { Valor t } \\
\text { (bootstrap) }\end{array}$ & $\mathrm{R}^{2}$ \\
\hline Masificación $\rightarrow$ Satisfacción & $-0.158^{* *}$ & & 3.585 & \\
\hline M. humana $\rightarrow$ M. turística & & $0.263^{* *}$ & 3.978 & \\
\hline M. espacial $\rightarrow$ M. turística & & $0.854^{* *}$ & 7.260 & \\
\hline Satisfacción & & & & 0.209 \\
\hline
\end{tabular}

Fuente: Elaboración propia con Datos de PLS

De los resultados anteriores se desprende que tanto la dimensión humana (peso $=0.263$ ) como la espacial $($ peso $=0.854$ ) contribuyen a la formación de la percepción de masificación turística de un destino, aunque es la dimensión espacial la que ejerce un mayor impacto sobre el mencionado constructo.

Los resultados también permiten concluir que la percepción de masificación turística influye en la satisfacción del turista de cruceros con la visita realizada al puerto de escala, ya que se ha encontrado una relación negativa y significativa entre ambos constructos $(\beta=-$ 0.119). La varianza explicada de la variable satisfacción, aunque supera el minimo recomendado del 10\% (Falk \& Miller, 1992), es bastante baja, lo que nos indica que existen otras variables adicionales que contribuyen en mayor medida a su formación.

\section{Conclusiones}

Desde una perspectiva cuantitativa la presente investigación cubre un gap importante en la literatura relacionada con la sostenibilidad en turismo y la experiencia del turista en el destino, al analizar, desde la óptica del crucerista, la percepción de masificación turística en sus viajes y su relación con la satisfacción obtenida en la visita.

Se comprueba que el turista de cruceros percibe tanto masificación humana como espacial, siendo la dimensión espacial la más relevante para este turista, quizás debido a que, por el tipo de turismo que practica, sabe que el número de personas con las que tendrá contacto en el destino (masificación humana) será elevado, por lo que es algo que, a priori, forma parte de sus expectativas. Por tanto, el crucerista se siente más molesto durante su visita por tener 
que hacer colas o por restricciones en su movilidad que por encontrarse con un gran número de personas.

Adicionalmente, se demuestra en la investigación que masificación turística y satisfacción son dos constructos relacionados, siendo la relación existente negativa y significativa. De ese modo, se corrobora en el presente estudio lo ya apuntado por Zehrer y Raich (2016), que entre ambas variables la relación es negativa significativa.

Estos resultados pueden ayudar a los gestores del destino a desarrollar estrategias para mejorar la movilidad, distribución y gestión del flujo de turistas en el destino. Adicionalmente, permiten tomar conciencia de la importancia de la sostenibilidad de un destino y de sus posibles repercusiones, haciéndose necesaria la adopción de prácticas de innovación sostenibles que ayuden a evitar consecuencias negativas derivadas de la masificación humana y espacial generada en un destino.

Sin duda, la pandemia COVID-19, sufrida durante el presente año, va a tener repercusiones positivas relacionadas con la sostenibilidad de los destinos. En relación al tema que nos ocupa, la necesidad de un distanciamiento social ayuda a reducir la masificación espacial y, en consecuencia, a reducir la percepción de masificación espacial de un destino. Ello tendrá una incidencia positiva sobre la satisfacción del turista con su visita e intenciones de comportamiento (intenciones de volver de nuevo al destino y de recomendarlo a otras personas).

\section{Referencias}

Arnberger, A., Aikoh, T., Eder, R., Shoji, Y. \& Mieno, T. (2010) How many people should be in the urban forest? A comparison of trail preferences of Vienna and Sapporo forest visitor segments, Urban Forestry \& Urban Greening, 9, pp. 215-225.

Bryon, J. \& Neuts, B. (2008) Crowding and the tourist experience in an urban environment: a structural equation modeling approach. Disponible en http://www.kuleuven.be/steunpunttoerisme/main/files/nieuwsbrief/oktober 2008/paperNVVS bart neuts.pdf.

Cheung, K. S., \& Li, L. H. (2019). Understanding visitor-resident relations in overtourism: Developing resilience for sustainable tourism. Journal of Sustainable Tourism, 27(8), 1197-1216.

CLIA (2019). Cruise Trends \& Industry Outlook. https://cruising.org/news-and-research//media/CLIA/Research/CLIA-2019-State-of-the-Industry.pdf. Consultado a fecha 22 abril 2020.

Díaz-Sauceda, J., Palau-Saumell, R., Forgas-Coll, S., Sánchez-García, J. (2015). Cross-border tourists' behavioral intentions: the Green Line of Nicosia, Cyprus. Tourism Geographies, 17(5), 758-779. 
Esser, A. H. (1972). Environment and the social sciences: Perspectives and applications. Washington, DC: American Psychological Association.

Falk, R., \& Miller, N. (1992). A Primer for Soft Modeling. Akron: University of Akron Press.

Gonzalez, V. M., Coromina, L., \& Galí, N. (2018). Overtourism: residents' perceptions of tourism impact as an indicator of resident social carrying capacity-case study of a Spanish heritage town. Tourism Review.

Hair Jr, J. F., Sarstedt, M., Ringle, C. M., Gudergan, S. P. (2017). Advanced issues in partial least squares structural equation modeling. Sage publications.

Jurado, E. N., Damian, I. M., Fernández-Morales, A. (2013). Carrying capacity model applied in coastal destinations. Annals of Tourism Research, 43, 1-19.

Kim, D., Lee, C. K., \& Sirgy, M. J. (2016). Examining the differential impact of human crowding versus spatial crowding on visitor satisfaction at a festival. Journal of Travel \& Tourism Marketing, 33(3), 293-312.

Kuščer, K., \& Mihalič, T. (2019). Residents' attitudes towards overtourism from the perspective of tourism impacts and cooperation-The case of Ljubljana. Sustainability, 11(6), 1823.

Li L., Zhang, J., Nian, S., Zhang, H. (2017). Tourists' perceptions of crowding, attractiveness, and satisfaction: a second-order structural model. Asia Pacific Journal of Tourism Research, 22(12), $1250-1260$.

Li, J. T., Kim, J., \& Lee, S. Y. (2009). An empirical examination of perceived retail crowding, emotions, and retail outcomes. The Service Industries Journal, 29(5), 635-652.

Noone, B. M., \& Mattila, A. S. (2009). Consumer reaction to crowding for extended service encounters. Managing Service Quality, 19, 31-41.

Popp, M. (2012). Positive and negative urban tourist crowding: Florence, Italy. Tourism Geographies, $14,50-72$.

Sanz-Blas, S., \& Carvajal, E. (2014). Cruise passengers' experiences in a Mediterranean port of call. The case study of Valencia. Ocean \& Coastal Management, 102, 307-316.

Sanz-Blas, S., Buzova, D., Schlesinger, W. (2019). The Sustainability of Cruise Tourism Onshore: The Impact of Crowding on Visitors' Satisfaction. Sustainability, 11(6), 1510.

Schmidt, D., \& Keating, J. (1979). Human crowding and personal control: An integration of the research. Psychological Bulletin, 86, 680-700.

Seraphin, H., Sheeran, P., \& Pilato, M. (2018). Over-tourism and the fall of Venice as a destination. Journal of Destination Marketing \& Management, 9, 374-376.

Zehrer, A., \& Raich, F. (2016). The impact of perceived crowding on customer satisfaction. Journal of Hospitality and Tourism Management, 29, 88-98. 